\title{
Antikörper hält Schübe in Schach
}

Auf eine lange anhaltende Wirksamkeit des monoklonalen Antikörpers Alemtuzumab in der MS-Therapie deuten Erweiterungen der beiden Phase-III-Studien CARE-MS I und CARE-MS II, in denen Alemtuzumab mit Interferon beta-1a verglichen wurde. An CARE-MS I nahmen 581 MS-Patienten teil, die bislang noch keine Basistherapie erhalten hatten, an CARE-MS II beteiligten sich 840 Patienten, bei denen unter der bisherigen Basistherapie erneut Schübe aufgetreten waren.

Nach zwei Jahren waren in den Gruppen mit Alemtuzumab $12 \mathrm{mg}$ i.v. deutlich mehr Patienten schubfrei als mit der Interferontherapie (78 vs. $59 \%$ in CAREMS I, 65 vs. $47 \%$ in CARE-MS II). Am Ende der Studien wurde allen Patienten eine Fortführung der Therapie mit dem Antikörper angeboten. Die Alemtuzumab-Patienten erhielten aber nur dann eine weitere Behandlungsphase, wenn es zu erneuten Schüben kam oder sich im
MRT neue Läsionen zeigten. Dies war im dritten Jahr nach Therapiebeginn nur bei jedem Fünften der Fall.

Von den 349 Patienten, die in CAREMS I von Beginn an Alemtuzumab erhalten hatten, waren nach dem Ende des dritten Jahres noch $67 \%$ schubfrei, von den 387 Patienten, die in CARE-MS II durchgehend den Antikörper bekommen hatten, waren es noch $55 \%$, sagte Dr. Edward Fox von der Universität in Round Rock, Texas. Bei den Patienten mit dreijähriger Therapie kam es auch nur selten zu einer Progression der Behinderungen. Bei den CARE-MS-I-Patienten war dies nach zwei Jahren bei $8 \%$ und nach drei Jahren bei $12 \%$ der Fall, bei den CARE-MS-II-Patienten lag der Anteil nach zwei Jahren bei $13 \%$ und nach drei Jahren bei $20 \%$.

Viel häufiger wurde das Gegenteil beobachtet - die Behinderungen gingen wieder zurück. Eine Verbesserung gemessen mit der Skala EDSS war nach drei
Jahren bei $40 \%$ in CARE-MS I und bei $45 \%$ in CARE-MS II zu beobachten, sagte Fox. Die MS ließ sich folglich beim größten Teil der Patienten auch im dritten Therapiejahr mit dem Antikörper gut kontrollieren.

In beiden CARE-MS-Studien war die Rate schwerer unerwünschter Wirkungen nicht höher als mit Interferon betala, allerdings traten vermehrt Autoimmunerkrankungen der Schilddrüse auf. Diese ließen sich jedoch durch ein Monitoringprogramm frühzeitig entdecken und gut kontrollieren, so Fox. Die Zulassung des neuen Medikamentes wird noch in diesem Jahr erwartet.

Thomas Müller, Springer Medizin

Scientific Platform Sessions, Multiple Sclerosis: Durable efficacy of Alemtuzumab in relapsing-remitting multiple sclerosis patients who participated in the CARE-MS studies. American Academy of Neurology, Annual Meeting, San Diego, 21.3.2013

\section{Bei Myopathien auf Befall der Atemmuskulatur achten}

Atemstörungen sind eine Symptomkomponente, die bei neuromuskulären Erkrankungen ein wichtiger differenzialdiagnostischer Hinweis sein können.

Ein 47-jähriger Mann klagte seit 18 Jahren über Schmerzen in allen großen Muskelpartien und zunehmende Muskelschwäche, schilderte Dr. Bertold Schrank, Deutsche Klinik für Diagnostik, Wiesbaden, eine Kasuistik beim Fallseminar „Neurologie im Dialog“. Das Aufstehen aus der Hocke war nur mit Gowers-Manöver möglich, andererseits erschienen die Oberarm- und Quadrizepsmuskulatur kräftig. Die Elektromyografie ergab myotone Serien im M. biceps femoris sowie in der Paravertebralmuskulatur. Die Thorakalmuskulatur wies in der Magnetresonanztomografie teilweise fettige Transformationen auf. Abgesehen von einem unspezifischen myopathischen Prozess bei axialer Myopathie (Bent-spine-Syndrom) konnte jedoch zunächst keine definitive Diagnose gestellt werden. Vermutet wurde zudem eine wesentliche psychosomatische Komponente. Allerdings nahm im Verlauf von neun Jahren die Vitalkapazität der Lunge stetig ab. Es traten schlafbezogene Atemstörungen (SBAS) auf, deren Symptome sich unter assistierter Beatmung nachts besserten.

\section{Proximale Muskelschwäche plus SABS - Verdacht auf M. Pompe}

Myopathien mit Befall der Atemmuskulatur bei noch gehfähigen Patienten sind vor allem die LGMD (limb girdle muscular dystrophy) 2I und die Glykogenose Typ II (Morbus Pompe), sagte Schrank. Bei der LGMD 2I bestehe keine enge Korrelation zwischen der Extremitätenund Atemmuskelbeteiligung. Morbus Pompe ist eine Glykogenspeicherkrankheit aufgrund eines vererbten Enzymmangels mit unter anderem proximaler Gliedergürtelschwäche. Bei erwachsenen Pompe-Patienten entwickelt sich die Symptomatik schleichend. Die frühe Diagnostik ist prognostisch relevant, weil seit 2006 mit Alglucosidase alfa (Myozyme ${ }^{\circledast}$ ) eine Enzymersatztherapie zur Verfügung steht, die das Fortschreiten der Krankheit aufhalten kann. Insofern sei das frühe Erkennen der Atemschwäche hilfreich und wichtig, betonte Professor Peter Young, Universitätsklinikum Münster. Die Patienten klagen über Kopfschmerzen, Erschöpfung, Abgeschlagenheit. Besonders im Liegen seien die paradoxe Atmung mit Einziehen des Bauches bei Inspiration sowie der abgeschwächte Hustenstoß erkennbar. Die schlafbezogene Atemstörung lässt sich im Schlaflabor sichern, das Vorliegen eines M. Pompe mit der Bestimmung der sauren alpha-Glukosidase in Lymphozyten.

Dr. Thomas Meißner, freier Medizinjournalist

\footnotetext{
Satellitensymposium „Neurologie im Dialog”, 57. Jahrestagung der Deutschen Gesellschaft für Klinische Neurophysiologie und Funktionelle Bildgebung (DGKN), Leipzig, 22.3.2013

Veranstalter: Genzyme $\mathrm{GmbH}$
} 